\title{
INJECTIVE MODULES OVER DOWN-UP ALGEBRAS
}

\author{
PAULA A. A. B. CARVALHO and CHRISTIAN LOMP \\ Departamento de Matemática, Faculdade de Ciências, Universidade do Porto, 4169-007 Porto, Portugal \\ and DILEK PUSAT-YILMAZ \\ Department of Mathematics, Izmir Institute of Technology, 35430 Gulbahce, Urla-Izmir, Turkey
}

\begin{abstract}
The purpose of this paper is to study finiteness conditions on injective hulls of simple modules over Noetherian down-up algebras. We will show that the Noetherian down-up algebras $A(\alpha, \beta, \gamma)$ which are fully bounded are precisely those which are module-finite over a central subalgebra. We show that injective hulls of simple $A(\alpha, \beta, \gamma)$-modules are locally Artinian provided the roots of $X^{2}-\alpha X-\beta$ are distinct roots of unity or both equal to 1 .
\end{abstract}

1. Injective hulls of simple modules over Noetherian rings. Injective modules are the building blocks in the theory of Noetherian rings. Matlis showed that any indecomposable injective module over a commutative Noetherian ring is isomorphic to the injective hull $E(R / P)$ of some prime factor ring of $R$. He also showed that any injective hull of a simple module is Artinian (see [15] and [16, Proposition 3]). Vamos characterized commutative rings $R$ whose injective hulls of simples are Artinian as those whose localization $R_{M}$ by maximal ideals are Noetherian ([21, Theorem 2]). Not necessarily commutative rings whose injective hulls of simples are Artinian were studied by Jans ([8]) and termed co-Noetherian. In [6] Dahlberg showed that injective hulls of simple modules over $U\left(\mathfrak{s l}_{2}\right)$ are locally Artinian. Since $U\left(\mathfrak{s l}_{2}\right)$ is an instance of a larger class of Noetherian domains, the down-up Algebras, introduced by Benkart and Roby in [2], Patrick F. Smith asked which Noetherian down-up algebras satisfy this finiteness condition on their injective hulls of simple modules. The purpose of this note is to give a partial answer to Smith's question. Recall that a module is called locally Artinian if every of its finitely generated submodules is Artinian.

1.1. In connection with the Jacobson Conjecture for Noetherian rings Jategaonkar showed in [9] (see also [5, 20]) that the injective hulls of simple modules are locally Artinian provided the ring $R$ is fully bounded Noetherian.

1.2. Consider the following property for a ring $A$ :

$(\diamond)$ Injective hulls of simple right $A$-modules are locally Artinian.

Property $(\diamond)$ is obviously equivalent to the condition, that all finitely generated essential extensions of simple right $A$-modules are Artinian. And in case $A$ is right Noetherian

This paper is dedicated to Patrick F. Smith - teacher and friend. 
property $(\diamond)$ is further equivalent to the class of semi-Artinian right $A$-modules, i.e. modules $M$ that are the union of their socle series, to be closed under essential extensions. In torsion theoretic terms, $A$ has property $(\diamond)$ if and only if the hereditary torsion theory generated by all simple right $A$-modules is stable.

1.3. Let us first explain, why every commutative Noetherian ring has property $(\diamond)$ without using Matlis result. The Artin-Rees Lemma says (in one of its versions) that any ideal $I$ of a commutative Noetherian ring $A$ has the Artin-Rees property, i.e. for any essential extension $N \subseteq M$ of finitely generated $A$-modules with $N I=0$, there exists $n>0$ such that $M I^{n}=0$. Thus $M$ has a finite filtration

$$
0 \subseteq M I^{n-1} \subseteq M I^{n-2} \subseteq \cdots \subseteq M I^{2} \subseteq M I \subseteq M
$$

such that each of its factors $M I^{k-1} / M I^{k}$ is a finitely generated $A / I$-module. If $N$ is a simple right $A$-module and $I=\operatorname{Ann}_{A}(N)$ then $A / I$ is a field, hence Artinian, and so any factor in the filtration of $M$ is Artinian, making $M$ an Artinian module.

A sufficient condition for $(\diamond)$ is therefore that right primitive ideals of $A$ have the Artin-Rees property and that primitive factor rings of $A$ are Artinian.

1.4. For simple Noetherian algebras, the above argument cannot be used due to the absence of non-zero proper ideals. However if $A$ is a (not necessarily commutative) semiprime Noetherian ring of Krull dimension one, then for any essential right ideal $I$ of $A$, the Krull dimension of $A / I$ is one lower than the Krull dimension of $A$, hence Artinian. For any extensions $E \subseteq M$ of a simple right $A$-module $E$ by a cyclic right $A$-module $M=A / I$, we have $E \simeq J / I$ with $J / I$ essential in $A / I$. Since pre-images of essential submodules are essential, also $J$ is essential in $A$. Thus $M / E \simeq A / J$ is Artinian and $M$ being an extension of the two Artinian modules $E$ and $M / E$ is also Artinian. Thus any semiprime Noetherian ring of Krull dimension one has property $(\diamond)$. This applies in particular to the first Weyl algebra $A_{1}(\mathbb{C})=\mathbb{C}[x][y ; \partial / \partial x]$.

1.5. Let $E \subseteq M$ be an essential extension of a simple right $A$-module $E$ by a Noetherian module $M$. Let $P=\operatorname{Ann}_{A}(E)$ be its annihilator. Suppose there exists a non-zero central element $x \in P \cap Z(A)$, i.e. $E x=0$. Denote by $f: M \rightarrow M$ the $A$ linear map $f(m)=m x$. Its kernel is $\operatorname{Ker}(f)=\operatorname{Ann}_{M}(x)$. By Fitting's Lemma there exists a number $n>0$ such that $\operatorname{Im}\left(f^{n}\right) \cap \operatorname{Ker}\left(f^{n}\right)=0$. Since $M$ is uniform and $E \subseteq \operatorname{Ker}\left(f^{n}\right)$ is non-zero, we have $\operatorname{Im}\left(f^{n}\right)=M x^{n}=0$. Hence we have again a finite filtration

$$
0 \subseteq \operatorname{Ker}(f)=\operatorname{Ann}_{M}(x) \subseteq \operatorname{Ker}\left(f^{2}\right) \subseteq \cdots \subseteq \operatorname{Ker}\left(f^{n-1}\right) \subseteq \operatorname{Ker}\left(f^{n}\right)=M
$$

whose factors $\operatorname{Ker}\left(f^{k}\right) / \operatorname{Ker}\left(f^{k-1}\right)$ are $A / A x$-modules and embed into $\operatorname{Ann}_{M}(x)$, because $f$ induces monomorphisms

$$
M / \operatorname{Ker}\left(f^{n-1}\right) \hookrightarrow \operatorname{Ker}\left(f^{n-1}\right) / \operatorname{Ker}\left(f^{n-2}\right) \hookrightarrow \cdots \hookrightarrow \operatorname{Ker}\left(f^{2}\right) / \operatorname{Ker}(f) \hookrightarrow \operatorname{Ker}(f) .
$$

Hence $M$ is Artinian if and only if $\operatorname{Ann}_{M}(x)=\operatorname{Ker}(f)$ is Artinian. The same argument also works for $x$ being a normal element. In this case $f$ is not $A$-linear anymore, but preserves submodules (see [10, Lemma 2]). 
1.6. The last subsection allow us now to state the following reduction of our problem, in case $A$ has a non-trivial centre.

PROPOSITION. The following statements are equivalent for a countably generated Noetherian algebra $A$ with Noetherian centre over an algebraically closed uncountable field $K$.

(a) Injective hulls of simple right A-modules are locally Artinian;

(b) Injective hulls of simple right $A / \mathfrak{m} A$-modules are locally Artinian for all maximal ideals $\mathfrak{m}$ of $Z(A)$.

Proof. $(a) \Rightarrow(b)$ is clear, since property $(\diamond)$ is inherited by factor rings.

$(b) \Rightarrow(a)$ : First note that the Proposition is void if $A$ has trivial centre $Z(A)=K$. Hence we will suppose $Z(A) \neq K$. Moreover note, that any countably generated algebra $A$ over an uncountable field $K$ has the endomorphism property (see [17, 9.1.7]). Hence the endomorphism ring of each simple right $A$-module $A$ is $\operatorname{End}_{A}(E) \simeq K$ as $K$ was supposed to be algebraically closed. Let $E$ be a simple right $A$-module and $M$ be a finitely generated essential extension of $E$. Denote $P=\operatorname{Ann}_{A}(E)$ and $\mathfrak{m}=P \cap Z(A)$ which is a maximal ideal of $Z(A)$ as the $A$-action on $E$ restricts to an $Z(A)$-action on $E$ which is not faithful as $\operatorname{End}_{A}(E)=K$ and $Z(A) \neq K$. As $A$ and $Z(A)$ are Noetherian, there exist central elements $x_{1}, \ldots, x_{k}$ that generate $\mathfrak{m}$. By $1.5 M$ is Artinian if and only if $M_{1}=\operatorname{Ann}_{M}\left(x_{1}\right)$ is Artinian. Applying the same argument again leads to $M$ Artinian if and only if $M_{2}=\operatorname{Ann}_{M_{1}}\left(x_{2}\right)=\operatorname{Ann}_{M}\left(\left\{x_{1}, x_{2}\right\}\right)$ Artinian. Iterating $k$ times leads to $M$ being Artinian if and only if $\operatorname{Ann}_{M}\left(\left\{x_{1}, \ldots, x_{k}\right\}\right)=\operatorname{Ann}_{M}(\mathfrak{m})$ being Artinian. Since $E \subseteq \operatorname{Ann}_{M}(\mathfrak{m})$ is an essential extension of $A / \mathfrak{m} A$-modules, with $\operatorname{Ann}_{M}(\mathfrak{m})$ being finitely generated, we get by hypothesis $(b)$, that $\operatorname{Ann}_{M}(\mathfrak{m})$ is Artinian.

1.7. Let $\mathfrak{h}$ be the three-dimensional Heisenberg algebra over $\mathbb{C}$ which is generated by $x, y, z$ with Lie algebra structure given by $[x, y]=z$ and $[x, z]=0=[y, z]$. Let $A=U(\mathfrak{h})$. Then $Z(A)=\mathbb{C}[z]$ and its maximal ideals are of the form $\mathfrak{m}_{\lambda}=\langle z-\lambda\rangle$, with $\lambda \in \mathbb{C}$. For $\lambda=0$, we have that $A / \mathfrak{m}_{0} A \simeq \mathbb{C}[x, y]$ is a commutative Noetherian domain and hence has property $(\diamond)$. For $\lambda \neq 0$, we have $A / \mathfrak{m}_{\lambda} A \simeq \mathbb{C}[x][y ; \partial / \partial x]$ is the first Weyl algebra, which is a Noetherian domain of Krull dimension 1 (see [17, 6.6.15]) and hence also has property $(\diamond)$ by 1.4 . Hence by Proposition 1.6 we have that $U(\mathfrak{h})$ has the property $(\diamond)$.

1.8. In contrast to the Heisenberg algebra, which is a nilpotent Lie algebra, Ian Musson showed that no non-nilpotent soluble finite-dimensional complex Lie algebra $\mathfrak{g}$ has property $(\diamond)$, i.e. there exists a non-Artinian finitely generated essential extension of a simple right $U(\mathfrak{g})$-module ([18]).

1.9. In [6] Dahlberg showed that $U\left(\mathfrak{s l}_{2}\right)$ has property $(\diamond)$. Since $U\left(\mathfrak{s l}_{2}\right)$ and $U(\mathfrak{h})$ are two instances of a larger class of Noetherian domains, the down-up Algebras, introduced by Benkart and Roby ([2]) we ask which Noetherian down-up algebras satisfy property $(\diamond)$.

In the following section we will recall the definition of down-up algebras and determine when they are fully bounded Noetherian. In the last section we show that some of the Noetherian down-up algebras of Krull dimension 2 have property $(\diamond)$. For simplicity, all algebras are considered to be algebras over the complex numbers $\mathbb{C}$. 
2. Fully bounded Noetherian down-up algebras. The down-up algebras form a three-parameter family of associative algebras. For any parameter set $(\alpha, \beta, \gamma) \in \mathbb{C}^{3}$ one defines a $\mathbb{C}$-algebra, denoted by $A=A(\alpha, \beta, \gamma)$, generated by two elements $u$ and $d$ subject to the relations

$$
\begin{aligned}
& d^{2} u=\alpha d u d+\beta u d^{2}+\gamma d \\
& d u^{2}=\alpha u d u+\beta u^{2} d+\gamma u
\end{aligned}
$$

2.1. Kirkman, Musson and Passman proved that $A$ is noetherian if and only if it is a domain if and only if $\beta \neq 0$ if and only if $\mathbb{C}[u d, d u]$ is a polynomial ring (see [11]).

2.2. Any Noetherian down-up algebra can be presented as generalized Weyl algebra. Let $R$ be a commutative ring and $\sigma$ and automorphism of $R$ and $x$ an element of $R$, the generalized Weyl algebra is the algebra $R(\sigma, x)$ generated over $R$ in two indeterminates $u, d$ subject to the relations: $u r=\sigma(r) u, d r=\sigma^{-1}(r) d$ for $r \in R$ and $u d=x, d u=\sigma^{-1}(x)$. In other words

$$
R(\sigma, x):=R\langle u, d\rangle /\left\langle u r-\sigma(r) u, d r-\sigma^{-1}(r) d, u d=x, d u=\sigma^{-1}(x) \forall r \in R\right\rangle .
$$

As shown in [11] and [13], if $\beta \neq 0$, then $A \simeq R(\sigma, x)$ where $R=\mathbb{C}[x, y], \sigma(x)=\frac{y-\alpha x-\gamma}{\beta}$ and $\sigma(y)=x$. The isomorphism maps $u d$ to $x$ and $d u$ to $y$. Kulkarni calls a Noetherian down-up algebra a down-up algebra at roots of unity if the associated automorphism $\sigma$ has finite order.

2.3. The centre of $R(\sigma, x)$ is generated by the fixed ring $R^{\sigma}$ and the elements $u^{m}, d^{m}$ where $m$ is the order of $\sigma$ or 0 if the order is infinite (see $\left.[13,2.0 .1]\right)$. Hence if $\sigma$ has finite order, by the above and Noether's Theorem, $R(\sigma, x)$ is finitely generated as a module over its centre. On the other hand if $\sigma$ has infinite order, then the centre of $R(\sigma, x)$ is equal to the fixed ring $R^{\sigma}$. Hence $R(\sigma, x)$ cannot be finitely generated over a central subalgebra since otherwise it would be also finitely generated as a module over $R$ which is impossible since $R(\sigma, x)=\bigoplus_{n \in \mathbb{Z}} A_{n}$ is $\mathbb{Z}$-graded with $A_{n}=R u^{n}$ and $A_{-n}=R d^{n}$ for $n>0$ and $A_{0}=R$. As any finitely generated $R$-submodule of $R(\sigma, x)$ is bounded and $A_{n} \neq 0$ for all $n, R(\sigma, x)$ is not finitely generated over $R$. Thus we proved:

LEMMA. $R(\sigma, x)$ is module-finite over a central subalgebra if and only if $\sigma$ has finite order.

2.4. Recall that a ring $R$ is called right (resp. left) bounded if every right (resp. left) essential ideal contains a non-zero two-sided ideal. $R$ is called right fully bounded Noetherian if it is right Noetherian and every prime factor ring is right bounded. As mentioned in the first section, fully bounded Noetherian rings have property $(\diamond)$. The considerations above deduce now the following characterization of down-up algebras at roots of unity.

THEOREM. The following statements are equivalent for a Noetherian down-up algebra $A=A(\alpha, \beta, \gamma)$ :

(1) $A$ is a down-up algebra at roots of unity;

(2) $A$ is module-finite over a central subalgebra; 
(3) A satisfies a polynomial identity;

(4) $A$ is fully bounded Noetherian;

(5) The roots of the polynomial $X^{2}-\alpha X-\beta$ are distinct roots of unity such that both are also different from 1 if $\gamma \neq 0$.

Proof. (1) $\Leftrightarrow$ (2) follows from Lemma 2.3. (2) $\Rightarrow$ (3) any module-finite algebra over a commutative subalgebra satisfies a polynomial identity (see for instance [17, 13.4.9]).

$(3) \Rightarrow(4)$ any Noetherian algebra that satisfies a polynomial identity is fully bounded Noetherian (see for instance $[\mathbf{1 7}, 13.6 .6]$ ).

(4) $\Rightarrow$ (1) we will show that $A$ is a down-up algebra at roots of unity.

Note that by [4, p. 287] any Noetherian down-up algebra $A$ can be embedded into the skew Laurent polynomial ring $R\left[z, z^{-1} ; \theta\right]$ where $R=\mathbb{C}[x, y], \theta(x)=y$ and $\theta(y)=\alpha y+\beta x+\gamma$. As a right $R$-module $R\left[z, z^{-1} ; \theta\right]$ is free on the basis $\left\{z^{n} \mid n \in \mathbb{Z}\right\}$ and the multiplication in $S$ is defined by $r z=z \theta(r)$ for $r \in R$. The embedding $\iota: A \rightarrow$ $R\left[z, z^{-1} ; \theta\right]$ is given by $\iota(d)=z^{-1}$ and $\iota(u)=x z$, so that $\iota(u d)=x$ and $\iota(d u)=y$. By the proof of $\left[\mathbf{4}\right.$, Lemma 1.2], $R\left[z, z^{-1} ; \theta\right]$ is the localization of $A$ by the Ore set $\left\{d^{n} \mid n \in \mathbb{N}\right\}$. By [3, 4.1.8] (or by [5, Proposition 1.5] and [12, Theorem 3.5]), if $A$ is fully bounded Noetherian, then also $A_{d}$, hence $R\left[z, z^{-1} ; \theta\right]$. By [3, Proposition 4.1.12], $\theta$ must have finite order. Since $A \simeq A^{\prime}=A\left(-\alpha \beta^{-1}, \beta^{-1},-\gamma \beta^{-1}\right)$ by [4, Lemma 4.1], also $\theta^{\prime}$ has finite order, where $\theta^{\prime}$ is the automorphism of $\mathbb{C}[x, y]$ defined analogously by $\theta^{\prime}(x)=y$ and $\theta^{\prime}(y)=\left(-\alpha \beta^{-1}\right) y+\left(-\beta^{-1}\right) x+\left(-\gamma \beta^{-1}\right)$. Denoting by $\tau$ the automorphism of $\mathbb{C}[x, y]$ which interchanges $x$ and $y$, we have that $\sigma=\tau \theta^{\prime} \tau$ has finite order, where $\sigma$ equals the automorphism that represents $A$ as a generalized Weyl algebra as in 2.2. Hence $A$ is a down-up algebra at roots of unity.

(1) $\Leftrightarrow(5)$ Let $r_{1}, r_{2}$ be the roots of the polynomial $X^{2}-\alpha X-\beta$ and let $\theta$ be the automorphism that defines the skew Laurent ring $R\left[z, z^{-1} ; \theta\right]$ as above, with $R=\mathbb{C}[x, y]$. Note that $\theta$ stabilizes the vector space $V$ spanned by $1, x$ and $y$. In [4, p. 288-289] a basis $1, w_{1}, w_{2}$ for $V$ had been found such that the matrix of $\theta$ with respect to this basis is in Jordan canonical form. Four cases had to be considered: if both roots $r_{1}$ and $r_{2}$ are different and also different from 1, then there exists such a basis such that $\theta\left(w_{i}\right)=r_{i} w_{i}$ for $i=1,2$. Hence $\theta$ has finite order if and only if both roots are roots of unity.

If $r_{1}=1$ and $r_{2} \neq 1$, then there exists a basis such that $\theta\left(w_{1}\right)=w_{1}+\gamma$ and $\theta\left(w_{2}\right)=$ $r_{2} w_{2}$. Hence $\theta$ has finite order if and only if $\gamma=0$ and $r_{2}$ is a root of unity.

If both roots are the same $r=r_{1}=r_{2}$ but different from 1 , then there exists a basis such that $\theta\left(w_{1}\right)=r w_{1}$ and $\theta\left(w_{2}\right)=r w_{2}+w_{1}$. Hence for any $n, \theta^{n}\left(w_{1}\right)=r^{n} w_{1}$ and $\theta^{n}\left(w_{2}\right)=r^{n} w_{2}+n r^{n-1} w_{1}$. Hence $\theta$ cannot have finite order. Similarly, if both roots are 1 , there exists a basis such that $\theta\left(w_{1}\right)=w_{1}+\gamma$ and $\theta\left(w_{2}\right)=w_{2}+w_{1}$ that implies that $\theta$ will not have finite order.

3. Non-primitive down-up algebras of Krull dimension two. A theorem of Bavula and Lenagan states, that the Krull dimension of $A=A(\alpha, \beta, \gamma)$ is 2 if and only if $\alpha+\beta=1$ and $\gamma \neq 0 \neq \beta$; otherwise the Krull dimension is 3 (see [1, Theorem 4.2]). Equivalently $A$ has Krull dimension 2 precisely if $\gamma, \beta \neq 0$ and 1 is a root of $X^{2}-$ $\alpha X-\beta$. We will focus in this section on those Down-Up algebras which are denoted by $A_{\eta}$ in $[4]: A_{\eta}:=A(1+\eta,-\eta, 1)$ for $\eta \in \mathbb{C}^{\times}=: \mathbb{C} \backslash\{0\}$. 
3.1. By [22, Theorem $1.3(\mathrm{~g})]$ the centre of the algebras $A_{\eta}$, with $\eta$ being a primitive $\mathrm{N}$-th root of unity different from 1 , is a polynomial ring $\mathbb{C}[\omega]$ in one variable, where $\omega$ is the element $\omega=z^{N}$ with $z=d u-u d+\frac{\gamma}{\eta-1}$. Note that if $\eta$ is not a root of unity, then the centre of $A_{\eta}$ is trivial. We will apply Proposition 1.6 to prove the following:

THEOREM. $A_{\eta}$ satisfies $(\diamond)$ if $\eta$ is a root of unity different from 1 .

Proof. Let $\eta$ be a primitive $N$ th root of unity different from 1 . We intend to use Proposition 1.6. As mentioned before $Z\left(A_{\eta}\right)=\mathbb{C}[\omega]$. The maximal ideals of $\mathbb{C}[\omega]$ are of the form $\langle\omega-c\rangle$ with $c \in \mathbb{C}$. By [19, Theorem 8.1(C1)] any ideal of the form $(\omega-c) A_{\eta}$ with $c \in \mathbb{C}^{\times}$is right primitive. Hence $A_{\eta} /(\omega-c) A_{\eta}$ is a right primitive Noetherian ring of Krull dimension 1 and hence has property $(\diamond)$ by 1.4. For $c=0$, let $B=A_{\eta} / \omega A_{\eta}$. We have that $\omega=z^{N}$ with $z=d u-u d+\frac{\gamma}{\eta-1}$. As $z$ is a normal element of $A_{\eta}$ it is also normal in $B$. By [19, Theorem 8.1(C1)] $z A_{\eta}$ is a primitive right ideal of $A_{\eta}$ and hence so is $z B$ as ideal of $B$. Thus $B / z B$ is a primitive Noetherian ring of Krull dimension 1 and has property $(\diamond)$ again by 1.4 . Given any essential extension $E \subseteq M$ of finitely generated $B$-modules, with $E$ being simple, we first note, that $z E=0$, since otherwise $E=z E=\cdots=z^{N} E=\omega E=0-$ a contradiction. Since $B / z B$ satisfies $(\diamond), \operatorname{Ann}_{M}(z)$ is Artinian and by [10, Lemma 2] $M$ is Artinian.

This shows that any factor $A_{\eta} / \mathfrak{m} A_{\eta}$ by a maximal ideal $\mathfrak{m}$ of $Z\left(A_{\eta}\right)$ has property $(\diamond)$. By Proposition 1.6 $A_{\eta}$ satisfies $(\diamond)$.

3.2. Summarizing Theorem 2.4 and Theorem 3.1 we have the following:

COROLlary. The injective hull of any simple right $A(\alpha, \beta, \gamma)$-module is locally Artinian, if the roots of $X^{2}-\alpha X-\beta$ are distinct roots of unity or both equal to one.

Proof. If the roots of $X^{2}-\alpha X-\beta$ are distinct roots of unity and also different from 1 if $\gamma \neq 0$, then $A=A(\alpha, \beta, \gamma)$ is fully bounded Noetherian by Theorem 2.4. A classical result by Schelter and Jategaonkar says that the injective hull of a simple right $R$-module over a left Noetherian right fully bounded Noetherian ring $R$ is locally Artinian (see for instance [7, 9.12] or [17, Proposition 6.4.14]).

Suppose $\gamma \neq 0$ and that one of the roots is 1 , then $A \simeq A_{\eta}$ and Theorem 3.1 shows that $A$ has property $(\diamond)$. In case both roots are 1 , then $\alpha=2$ and $\beta=-1$. Since $A_{1}=A(2,-1,1)=U\left(\mathfrak{s l}_{2}\right)$ and $A(2,-1,0)=U(\mathfrak{h})$ those algebras have property $(\diamond)$ by [6] and 1.7 .

3.3. We were unable to find an example of a Noetherian down-up algebra that does not satisfy $(\diamond)$.

AcKnowledgements. Special thanks to Patrick Smith who suggested the topic at the X. Antalya Algebra Days to the first author. The first two authors were partially supported by Centro de Matemática da Universidade do Porto (CMUP), financed by FCT (Portugal) through the programs POCTI (Programa Operacional Ciência, Tecnologia, Inovação) and POSI (Programa Operacional Sociedade da Informação), with national and European community structural funds. The third author would like to thank TUBITAK for financial support from project numbered 107T709. Parts of this research have been carried out during the second author's visit to the Max-Planck 
Institut für Mathematik in Bonn. He would like to thank the institute for the interesting atmosphere and the excellent working conditions.

\section{REFERENCES}

1. V. V. Bavula and T. H. Lenagan, Generalized Weyl algebras are tensor Krull minimal, J. Algebra 239(1) (2001), 93-111.

2. G. Benkart and T. Roby, Down-up algebras, J. Algebra 209(1) (1998), 305-344.

3. P. A. A. B. Carvalho, On the prime spectra of some Noetherian rings, $\mathrm{PhD}$ Thesis (University of Glasgow, 1998).

4. P. A. A. B. Carvalho and I. M. Musson, Down-up algebras and their representation theory, J. Algebra 228 (2000), 286-310.

5. G. Cauchon, Anneaux de polynòmes essentiellement borns, in Ring theory (Proc. Antwerp Conf. (NATO Adv. Study Inst.), University of Antwerp, Antwerp, 1978), Lecture Notes in Pure and Applied Mathematics, vol. 51 (F. van Oystaeyen, Editor) (Dekker, 1979), 27-42.

6. R. L. Dahlberg, Injective hulls of simple sl $(2$, C) modules are locally Artinian, Proc. Amer. Math. Soc. 107(1) (1989), 35-37.

7. K. R. Goodearl and R. B. Warfield, Jr., An introduction to noncommutative Noetherian rings, London Mathematical Society Student Texts 16. (Cambridge University Press, Cambridge, 1989).

8. J. P. Jans, On co-Noetherian rings, J. Lond. Math. Soc. 1(2) (1969), 588-590.

9. A. V. Jategaonkar, Jacobson's conjecture and modules over fully bounded Noetherian rings, J. Algebra 30 (1974), 103-121.

10. A. V. Jategaonkar, in Certain injectives are Artinian. in Noncommutative ring theory (Internat. Conf., Kent State University, Kent Ohio, 1975). Lecture Notes in Mathematics, vol. 545 (Springer, Berlin, 1976) 128-139.

11. E. Kirkman, I. M. Musson and D. S. Passman, Noetherian down-up algebras, Proc. Amer. Math. Soc. 127 (11) (1999), 3161-3167.

12. G. Krause, On fully left bounded left Noetherian Rings, J. Algebra 23 (1972), 88-99.

13. R. S. Kulkarni, Down-up algebras and their Representations, J. Algebra 245 (2001), 431-462.

14. R. S. Kulkarni, Down-up algebras at roots of unity, Proc. Amer. Math. Soc. 136 (10) (2008), 3375-3382.

15. E. Matlis, Injective modules over Noetherian rings, Pac. J. Math. 8 (1958), 511-528.

16. E. Matlis, Modules with descending chain condition, Trans. Amer. Math. Soc. 97(3) (1960), 495-508.

17. J. C. McConnell and J. C. Robson, Noncommutative Noetherian rings, Grad. Stud. Math. 30, AMS (2000).

18. I. M. Musson, Some examples of modules over Noetherian rings, Glasgow Math. J. 23 (1982), 9-13.

19. I. Praton, Primitive ideals in Noetherian down-up algebras, Commun. Algebra 32(2) (2004), 443-471.

20. W. Schelter, Essential extensions and intersection theorems, Proc. Amer. Math. Soc. 53(2) (1975), 328-330.

21. P. Vamos, The dual of the notion of "finitely generated", J. Lond. Math. Soc. 43 (1968), 643-646.

22. K. Zhao, Centers of down-up algebras, J. Algebra 214 (1999), 103-121. 